Teil 1

Rechtliche Rabmenbedingungen der Kreativität im Wandel 



\title{
Identifying Tipping Point(s): Wendepunkte bei der Verabschiedung der DSM-Richtlinie
}

\author{
Amélie Heldt"
}

Hat der Gesetzgeber die Pflicht, auf Entwicklungen seiner Zeit zu reagieren und welche Wendepunkte - sogenannte Tipping Points - haben zur Novellierung der EU-Urheberrechtsrichtlinie geführt? ${ }^{\text {I }}$ In diesem Beitrag geht es im Schwerpunkt um eine Analyse des Prozesses der Verabschiedung der DSM-Richtlinie anhand des Konzepts der Tipping Points. Dafür wird zunächst auf die Definition des Tipping Point-Konzepts und auf das Verhältnis zwischen der Öffentlichkeit und dem allgemeinen Gesetzgebungsverfahren eingegangen, um danach eine Antwort auf die Frage zu geben, welche Rolle die Öffentlichkeit im Prozess der Verabschiedung der finalen Version, insbesondere des nunmehr Art. 17 der DSM-RL, gespielt hat. Sowohl die Emergenz neuer privater Akteure durch den digitalen Wandel als auch die Debatte um Upload-Filter sind dabei hervorzuheben.

\section{Definition des Tipping Points}

Der Journalist Gladwell beschreibt den Begriff des Tipping Points wie folgt: „An einem Tipping Point ist für einen Moment lang alles offen Veränderungen, die in einem solchen Moment, an einem solchen Punkt vorgenommen werden, können ungeheuer wirkungsvoll sein. " ${ }^{2}$ und allgemein definiert man ihn als „kritischen Punkt in einer Situation, einem

* Ass. iur. Amélie Heldt, maître en droit ist Juristin und forscht als wissenschaftliche Mitarbeiterin am Leibniz-Institut für Medienforschung | Hans-Bredow-Institut in Hamburg zur Transformation der öffentlichen Kommunikation. Ihre Forschungsschwerpunkte sind Verfassungsrecht und Media Governance.

1 Die Autorin möchte Prof. Dr. Martin Kretschmer und den Teilnehmer:innen der Fachtagung Urheberrecht, sowie Prof. Dr. Wolfgang Schulz und Dr. Stephan Dreyer für wertvolles Feedback danken. Danke an Johanna Stelling, Rike Heyer und Carlotta Siegel für ihre Unterstützung.

2 Gladwell, Der Tipping Point, 266. 
Prozess oder einem System, über den hinaus eine signifikante und oft unaufhaltsame Wirkung oder Veränderung stattfindet.“3

Im Kontext von politischen Entscheidungen können Tipping Points Wendepunkte in Agenda-Setting-Prozessen darstellen, wie aus der PolicyForschung von Jones und Baumgartner hervorgeht. Sie entwickelten die Theorie des Punctuated-Equilibrium-Process, um Richtungswechsel in der US-Politik zu analysieren und zu erklären. ${ }^{4}$ Laut ihnen kommt es zu Änderungen, wenn Machtverhältnisse unterschiedlicher Interessengruppen an einem politischen Thema aus ihrem bisherigen Gleichgewicht geraten. ${ }^{5}$ Dabei ist keine institutionalisierte Form der Macht erforderlich, grundsätzlich kann es sich um Interessensgruppen handeln, die nicht am Gesetzgebungsverfahren beteiligt werden müssen. Auf dieser Prämisse baut auch das Threshold-Modell von Granovetter auf: Demnach kann eine kritische Menge von Individuen, die sich einer Sache gegenüber verhalten, zu einem Tipping Point führen, wenn ihre Ablehnung einen Schwellenwert erreicht. ${ }^{6}$ Wenn die kollektive Ablehnung signifikant zunimmt, wirkt es sich auf die bisherigen Machtverhältnisse aus - es entsteht möglicherweise ein neues Equilibrium. Diese Änderungen der Akzeptanz hat Noelle-Neumann in ihrer Theorie der Schweigespirale erklärt: Die öffentliche Meinung ist die „Meinung, die man öffentlich äußern kann, ohne sich zu isolieren." Wenn sie sich ändert, kann ein Änderungsdruck auf die sozialen Normen entstehen - auch für den Gesetzgeber. ${ }^{8}$ Folglich handelt es sich bei einem Tipping Point um eine Stufe des politischen Aushandlungsprozesses, die für die beteiligten Stakeholder-Gruppen als wichtig und unumkehrbar identifiziert wird.

3 Definition v. Merriam-Webster, freie Übersetzung d. Verf.: „critical point in a situation, process, or system beyond which a significant and often unstoppable effect or change takes place”.

4 True/Jones/Baumgartner, Punctuated-Equilibrium Theory, in: Sabatier, Theories of the Policy Process, $155 \mathrm{f}$.

5 Baumgartner/Jones, Agendas and Instability in American Politics, 278.

6 Granovetter, American journal of sociology 1978, 1420; Dan Wood/Doan, Am J Political Science 2003, 640, 641.

7 Noelle-Neumann, Öffentliche Meinung, 91.

8 Noelle-Neumann, Öffentliche Meinung, 178 f.; Fraenkel, Zeitschrift für Politik 1963, 309, 318; Lüdemann, Gesetzgebung als Entscheidungsprozess, $32 \mathrm{f}$. 


\section{Gesetzgebungsinitiative und Öffentlichkeit}

Um zu erkennen, wann ein Tipping Point eingetreten ist und zu einer Änderung des geltenden Rechts führt, ist es wichtig zu identifizieren, welche Akteure am Gesetzgebungsverfahren beteiligt sind und welche Rolle die Öffentlichkeit in Agenda-Setting-Prozessen innehat.

\subsection{Im Gesetzgebungsverfahren}

\subsubsection{Initiativrecht}

Gemäß dem Demokratieprinzip aus Art. 20 Abs. 2 S. 1 und 2 GG muss die legislative Gewalt vom Volk ausgehen und durch Wahlen und Abstimmungen legitimiert sein („Alle Staatsgewalt geht vom Volke aus.“). Die Zuständigkeit des deutschen Gesetzgebers ist in Art. 70 f. GG geregelt und teilt sich auf die Länder und den Bund auf. Auf Bundesebene ist der Bundestag als Organ für die Gesetzgebung zuständig. Es besteht kein exklusives Initiativrecht eines Organs zu Gesetzesvorhaben: Gemäß Art. 76 Abs. 1 GG können Gesetzvorlagen durch die Bundesregierung, aus der Mitte des Bundestages oder durch den Bundesrat eingebracht werden.

Auf EU-Ebene kann die Europäische Union gemäß Art. 2 Abs. 1 AEUV in solchen Bereichen tätig werden, in denen die Mitgliedstaaten ihr die Kompetenz übertragen haben. Das ordentliche Gesetzgebungsverfahren der EU ist in Art. 289 Abs. 1 i.V.m. Art. 294 AEUV geregelt und beginnt mit einem Vorschlag der EU-Kommission. Dieser wird dem Europäischen Parlament und dem Rat unterbreitet, woraufhin beide Organe sich über eine Fassung einigen müssen, bevor der letzte Entwurf gemäß Art. 294 Abs. 13 AEUV dem Europäischen Parlament zur Abstimmung vorliegt. Die demokratische Legitimierung dieser beiden Organe findet mittel- und unmittelbar statt: Der Rat besteht aus Staatsoberhäuptern der Mitgliedstaaten, die jeweils durch nationale Wahlen legitimiert sind. Das Europäische Parlament wird hingegen unmittelbar durch die Bürger der Europäischen Union (Art. 14 Abs. 3 EUV) gewählt. Darüber hinaus kann die Kompetenzübertragung der Mitgliedstaaten auf die EU durch die jeweiligen nationalen Parlamente auch als Form der mittelbaren Legitimierung angesehen werden.

So sind sowohl der deutsche als auch der europäische Gesetzgeber zum Erlass von Rechtsakten ermächtigt, nicht für einzelne Gesetzesvorhaben, sondern für einen im Vorhinein festgelegten Zeitraum (Wahlperiode) und ohne formelle Einwirkungsmöglichkeiten des Volkes ab dem Zeitpunkt, 
ab dem die gewählten Abgeordneten zu ihrer konstituierenden Sitzung zusammengekommen sind (bspw. $\$ 1$ GOBT).

\subsubsection{Initiativpflicht?}

Kann ein Tipping Point zu einer Handlungspflicht des Gesetzgebers führen? Im deutschen Verfassungsrecht gilt die Einschätzungsprärogative des Gesetzgebers, d.h. es bleibt ihm überlassen, ein Gesetzesvorhaben einzuleiten. Im Regelfall geschieht dies aufgrund des politischen Gestaltungswillen der gewählten Mehrheit. Dabei ist der Gesetzgeber gemäß Art. 20 Abs. 3 GG an die verfassungsmäßige Ordnung gebunden, d.h. Gesetze müssen gemäß dem Prinzip der Verhältnismäßigkeit (u.a.) geeignet und erforderlich sein. Das Übermaßverbot regelt jedoch nicht solche Situationen, in denen der Gesetzgeber tätig werden sollte, es aber nicht tut.

Nach dem Prinzip des Untermaßverbots kann für den Staat die Pflicht entstehen, aktiv zu werden. Das Untermaßverbot wird aus dem Rechtsstaatsprinzip gemäß Art. 20 Abs. 3 GG in Verbindung mit einzelnen Grundrechten abgeleitet. ${ }^{9}$ Das Untermaßverbot erklärt das Bundesverfassungsgericht wie folgt: „Notwendig ist ein - unter Berücksichtigung entgegenstehender Rechtsgüter - angemessener Schutz; entscheidend ist, dass er als solcher wirksam ist. Die Vorkehrungen, die der Gesetzgeber trifft, müssen für einen angemessenen und wirksamen Schutz ausreichend sein und zudem auf sorgfältigen Tatsachenermittlungen und vertretbaren Einschätzungen beruhen. "10 Dem Gesetzgeber steht weiterhin ein „Einschätzungs-, Wertungs- und Gestaltungsspielraum“ zu, d. h. es gibt keine Initiativpflicht, solange er einen Mindeststandard einhält, der eine Verletzung seiner Schutzpflichten vorbeugt. ${ }^{11}$

9 Grzeszick, in Maunz/Dürig, Rn. 126.

10 BVerfG, Urt. v. 28.05.1993 - 2 BvF 2/90, 2 BvF 4/92, 2 BvF 5/92 - Schwangerschaftsabbruch II.

11 BVerfG, Urt. v. 28.05.1993 - 2 BvF 2/90, 2 BvF 4/92, 2 BvF 5/92 - Neuregelung des Schwangerschaftsabbruchs. 


\subsection{Rolle der Öffentlichkeit}

Kommt es zu Änderungen in der Gesellschaft, gefolgt von einer Reaktion des Gesetzgebers, ist die Wahl des Parlaments nicht die einzige Verbindung zwischen dem Volk und der Legislative, sondern gesellschaftlicher Konsens wird in der Öffentlichkeit ausgehandelt. Ein Tipping Point kann u.a. an einer überwiegenden Haltung in der Bevölkerung erkannt werden. Modelle wie das Threshold-Modell von Wood und Doan beruhen auf der Annahme, durch die Summierung individueller Ablehnungsreaktionen ließe sich ein Schwellenwert ermitteln, ab dem die öffentliche Meinung sich wandelt - also ein Tipping Point in puncto gesellschaftlicher Akzeptanz. ${ }^{12}$ In ihrer Anwendung des Threshold-Modells in der US-Gesellschaft weisen sie auch auf die Rolle der Öffentlichkeit hin.

Der Begriff der Öffentlichkeit ist eines der meist diskutierten Konzepte der Sozialwissenschaft und kann hier nicht in extenso dargestellt werden. Das Verständnis und die Auslegung des Demokratieprinzips beruht in Deutschland auf der Theorie der deliberativen Demokratie einschließlich der ausgeführten Form der Öffentlichkeit von Habermas. ${ }^{13}$ Auch der vorliegende Aufsatz beruft sich auf die Theorie von Habermas und das DreiEbenen-Modell von Gerhards und Neidhardt. ${ }^{14}$ Laut Gerhards beschreibt Öffentlichkeit den „kommunikativen Bereich, in dem alle Bürger mit Argumenten öffentliche Belange diskutieren, an deren Ende eine öffentliche Meinung steht, die die Grundlage politischer Entscheidungen bildet" ${ }^{15} \mathrm{Im}$ klassischen Drei-Ebenen-Modell wird zwischen drei Ebenen der Öffentlichkeit und ihrer Institutionalisierung unterschieden: Einfache, mittlere und komplexe Öffentlichkeit. ${ }^{16}$ Einfache Öffentlichkeit entsteht bei persönlichen Begegnungen; mittlere, wenn der Personenkreis aufgrund der (erhöhten) Zahl der Beteiligten nicht mehr als persönlich bezeichnet werden kann; und komplexe Öffentlichkeit entsteht durch massenmediale Medien. Folglich wird eine Meinung als öffentliche Meinung betrachtet, wenn sie über die sogenannten Encounter- und Stammtisch-Öffentlichkeiten hinaus wahrgenommen und in der massenmedialen Öffentlichkeit ge-

12 Dan Wood/Doan, Am J Political Science 2003, 640, 652.

13 inter alia Pfetsch u. a., Publizistik 2018, 477, 483.

14 Habermas, Strukturwandel der Öffentlichkeit; Gerhards/Neidhardt, Strukturen und Funktionen moderner Öffentlichkeit.

15 Gerhards, in: Jarren/Sarnicelli/Saxer, Politische Kommunikation in der demokratischen Gesellschaft: ein Handbuch mit Lexikonteil, 268, 270.

16 Gerhards/Neidhardt, Strukturen und Funktionen moderner Öffentlichkeit, 19-26. 
spiegelt wird. ${ }^{17}$ Der Tipping Point als Richtungswechsel einer Gesellschaft zu einer Frage kann sich in Form einer öffentlichen Meinung manifestieren.

Die Staatslehre kennt verschiedene Attributionen der Öffentlichkeit, u. a. die umstrittene Akklamationsfunktion nach Carl Schmitt. ${ }^{18}$ Die Rechtsprechung des Bundesverfassungsgerichts beruht auf dem Habermas'schen Konzept der Öffentlichkeit, also auf einem Idealmodell des rationalen Diskurses in einer deliberativen Demokratie, insbesondere bei der Auslegung der Kommunikationsfreiheiten und der Konkretisierung der individuellen und kollektiven Meinungsbildung. ${ }^{19}$ Die zentralen Funktionen der Öffentlichkeit werden durch Grundrechte geschützt, d. h. Meinungs- und Informationsfreiheit, Versammlungsfreiheit und Presse-/Medienfreiheiten. Für die vorliegende Untersuchung ist vor allem wichtig, dass die Differenzierung zwischen privat einerseits und öffentlich (im Sinne von staatlich) andererseits weiterhin stattfindet, aber dass im Internet private Akteure eine wichtige Rolle spielen, deren Machtzunahme ein Tipping Point für den Gesetzgeber darstellen könnte.

Die Beteiligung der Öffentlichkeit kann als Voraussetzung im Gesetzgebungsverfahren vorgesehen sein und bezweckt durch die Zugänglichkeit von Informationen Qualitätssteigerung und Akzeptanzförderung. ${ }^{20}$ Die Beteiligung der Öffentlichkeit kann als Verständigung aller Betroffenen gestaltet werden und betrifft größtenteils Verfahren, die bereits von der öffentlichen Hand initiiert sind. Über Volksabstimmungen werden bereits entworfene Gesetze den Bürgern zur Abstimmung vorgelegt (sogenanntes Plebiszit). ${ }^{21}$ Direkte Formen der Bürgerbeteiligung sind auf Bundesebene nur bei der Neugliederung des Bundesgebietes gemäß Art. 29 GG vorgesehen, wohingegen Bürgerbegehren und Bürgerentscheide in den Verfassungen der Länder vorkommen. Auf EU-Ebene kann gemäß Art. 11 Abs. 4 EUV eine Europäische Bürgerinitiative entstehen, wenn ein Quorum von 1 Millionen EU-Bürger die EU-Kommission dazu auffordert, einen Vorschlag zur Umsetzung der EU-Verträge zu unterbreiten. Gemäß Art. 24 Abs. 2 i.V.m. Art. 277 AEUV hat jeder EU-Bürger ein Petitionsrecht, d. h. sie können sich in Angelegenheiten, die in die Tätigkeitsbereiche der Uni-

17 Auch wenn diese Konzepte vor der Digitalisierung entstanden sind, lassen sie sich entsprechend auf heutige Formen der Öffentlichkeit anwenden.

18 Schmitt, Verfassungslehre.

19 Hoffmann-Riem/Schulz, in: Jarren/Sarcinelli/Saxer, Politische Kommunikation in der demokratischen Gesellschaft: Ein Handbuch mit Lexikonteil, 154.

20 Schink, in Spannowsky/Uechtritz, $\mathbb{3}$ Rn. 3.

21 Weber, Volksabstimmung, in Creifelds kompakt, Rechtswörterbuch. 
on fallen und die ihn oder sie unmittelbar betreffen, an das Europäische Parlament wenden und es auffordern, ein besonderes Gesetzgebungsverfahren einzuleiten. Diese Formen der formalisierten Öffentlichkeitsbeteiligungen sind für das Demokratieprinzip unverzichtbar und können vereinzelt von Bürger:innen genutzt werden, um den Gesetzgeber zum Handeln zu veranlassen. Es handelt sich allerdings nicht zwangsläufig um Tipping Points, denn der Wendepunkt der öffentlichen Meinung kann mit einem Volksbegehren einhergehen, aber sie können theoretisch losgelöst voneinander stattfinden.

Um die informelle Rolle der Öffentlichkeit zu erfassen, gelangt man zurück zum Ausgangspunkt der Machtverhältnisse unterschiedlicher Interessengruppen innerhalb eines politischen Aushandlungsprozesses ${ }^{22}$ und der eventuellen Notwendigkeit einer Anpassung der Gesetzeslage. Grundsätzlich gibt es in allen Gesellschaften soziale Normen, die teils in der Form von Rechtsnormen normiert werden: ${ }^{23}$ Verhaltensregeln können individuell oder sozial sein, und soziale Verhaltensregeln sind normiert oder nicht. Zahlreiche gesellschaftliche Abläufe bedürfen keiner Rechtsnorm, weil die soziale Norm innerhalb des „als Einheit umgrenzbaren sozialen Gebildes“24 der Gesellschaft bekannt und anerkannt ist, somit auch der gesellschaftliche Druck, sie zu befolgen, ausreicht. Popitz beschreibt den Übergang von Sittennormen zu Rechtsnormen als das Überschreiten eines bestimmten Grad der Verfestigung (Institutionalisierung), ${ }^{25}$ doch wann dieser Schwellenwert erreicht wird, beantwortet er nicht.

Die Wechselwirkung zwischen sozialer Norm und Rechtsnorm ist komplex: Einerseits soll der Gesetzgeber nicht auf jede Änderung der öffentlichen Meinung reagieren (das Recht darf „eine Barriere gegen den bloßen Zeitgeist" sein, so Noelle-Neumann ${ }^{26}$ ), andererseits soll er sich nicht ohne Grund gegen gesellschaftliche Entwicklungen sperren. Dieses Bedürfnis einer Übertragung in gesetzliche Regelungen kann durch die Öffentlichkeit gespiegelt, formuliert und weitergetragen werden, die mediale Öffentlichkeit kann als Legitimationsmedium dienen. ${ }^{27}$

22 Siehe Jones/Baumgartner, Fn. 4.

23 Popitz, Die normative Konstruktion von Gesellschaft, 70.

24 Popitz, Die normative Konstruktion von Gesellschaft, 69.

25 Popitz, Die normative Konstruktion von Gesellschaft, 31.

26 Noelle-Neumann, Öffentliche Meinung, 184.

27 Vgl. BVerfG, Urt. v. 28.02.1961 - 2 BvG 2/60, BVerfGE 12, 205, 260 - Rundfunk I. 


\section{Tipping Points im Urheberrecht}

Überträgt man die bis hier dargestellten Gedanken auf die EU-Urheberrechtsrichtlinie, kristallisieren sich zwei mögliche Tipping Points heraus: Zunächst der erste Vorschlag der Richtliniennovellierung als Reaktion auf gesellschaftliche und technische Entwicklungen. Später die Änderung des Art. 17, welchem eine öffentliche Debatte um die Einführung von UploadFiltern vorangegangen war.

Der Schutz des Urheberrechts und seiner verwandten Schutzrechte ist

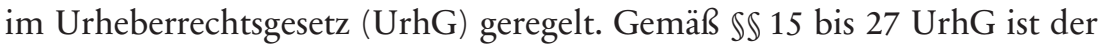
Urheber geschützt und kann grundsätzlich über die Nutzung und die Verwertung seines Werkes verfügen. Das starke Schöpferprinzip dient dem Urheber auf individueller Ebene und der Gesellschaft auf kollektiver Ebene, um Kultur zu fördern und gleichzeitig zugänglich $\mathrm{zu}$ machen (beispielsweise durch Ausnahmen in Form von gesetzlich erlaubten Nutzungen, $\mathbb{S} \mathbb{S} 44 \mathrm{a}-53 \mathrm{a}$ UrhG). Das Urheberrecht soll die widerstreitenden Interessen von Urhebern, Verwertern und Gesellschaft an dem Werk in Ausgleich bringen. Auf EU-Ebene wurde 2001 die Richtlinie zur Harmonisierung bestimmter Aspekte des Urheberrechts und der verwandten Schutzrechte in der Informationsgesellschaft verabschiedet, die 2003 ins deutsche Recht umgesetzt wurde.

\subsection{Neue Akteure als Game-Changer}

Durch das Internet sind traditionelle Massenmedien nicht mehr die einzigen Stimmen der Öffentlichkeit: Mithilfe sozialer Medien können Internetnutzer:innen einen Rezipientenkreis erreichen, der über ihre persönliche Sphäre hinausgeht. ${ }^{28}$ Die Digitalisierung hat auch Änderungen für das geistige Eigentum und dessen Verwertung gebracht, sowohl auf struktureller als auch auf inhaltlicher Ebene.

Strukturell sind neue Akteure auf den Plan getreten, die den Zugang, die Vervielfältigung und die Verbreitung von Inhalten ermöglichen, vereinfachen und beschleunigen. Das Geschäftsmodell von Plattformen beruht darauf, nutzergenerierte Inhalte in den Mittelpunkt zu stellen, auch Inhalte von Dritten, die urheberrechtlich geschützt sind. Zwar gibt es die Möglichkeit, Rechteverletzungen zu melden und Videos zu monetarisie- 
ren, aber diese Optionen wurden bisher für unzureichend erachtet. ${ }^{29}$ Auslöser für die Reform war nicht die Ermöglichung der Zurverfügungstellung von geschützten Werken durch Plattformen - also der technische $\mathrm{Zu}$ gang -, sondern der sogenannte „Value Gap“, d. h. die Diskrepanz zwischen den werbefinanzierten Einnahmen der Plattformen und der Ausschüttung an die Rechteinhaber. ${ }^{30}$ Abgesehen von den neuen Akteuren haben neue Formate und Inhalte auch zu strukturellen Änderungen geführt.

\subsection{Gesetzgebungsverfahren für das Urheberrecht im digitalen Binnenmarkt}

Die grundsätzliche Entwicklung des digitalen Binnenmarkts, neue Akteure und Geschäftsmodelle sowie die öffentliche Debatte diesbezüglich haben dazu geführt, dass auf EU-Ebene die Novellierung der Urheberrechtsrichtlinie angestrengt wurde. Alle Aspekte der Debatte rund um die neue EUUrheberrechtsrichtlinie können und sollen an dieser Stelle nicht wiedergegeben werden. ${ }^{31}$ Zusammengefasst ging es bei der Schaffung des Art. 17 der Richtlinie um den Schutz der Verwertungsmöglichkeiten von geschützten Werken vor einer - für die Hosting-Plattformen und somit auch deren Nutzer - kostenfreien Nutzung der Werke, also um den Schutz des Inhabers der Vergütungsansprüche aus dem UrhG. ${ }^{32}$ Um die Haftungsprivilegierung der Plattformen und die Ansprüche aus dem urheberrechtlichen Verwertungsrecht in Einklang zu bringen, enthielt der Vorschlag der EU-Kommission 2016 in (Ex-)Art. $13^{33}$ die Pflicht für „Diensteanbieter der Informationsgesellschaft“, Maßnahmen zur Vermeidung von Rechteverletzungen zu ergreifen, „wie beispielsweise wirksame Inhaltserkennungstechniken. “34 Konkreter war in (Ex-)Art. 13 Nr. 3 geregelt, dass Diensteanbieter und Mitgliedstaaten diese Maßnahmen wie „geeignete und angemessene

29 Vgl. den jahrelangen Streit zwischen der GEMA und YouTube.

30 Gielen/Tiessen, EuZW 2019, 639, 640 zur Entstehung der Reform.

31 Richtlinie (EU) 2019/790 des Europäischen Parlaments und des Rates v. 17.04.2019 über das Urheberrecht und die verwandten Schutzrechte im digitalen Binnenmarkt und zur Änderung der Richtlinien 96/9/EG und 2001/29/EG: https:/ /eur-lex.europa.eu/legal-content/DE/TXT/HTML/?uri=CELEX:32019L0790\&qid=1 579079069995\&from=DE, (zuletzt abgerufen am 15.5.2020).

32 Siehe Titel IV der Richtlinie: Maßnahmen zur Schaffung eines funktionsfähigen Marktes für den Urheberrechtsschutz.

33 Nunmehr Art. 17.

$34 \operatorname{COM(2016)} 593$ final, https://eur-lex.europa.eu/legal-content/DE/TXT/HTML/?uri $=$ CELEX:52016PC0593\&qid $=1579103662332 \&$ from $=$ DE (zuletzt abgerufen am 15.1.2020). 
Inhalterkennungstechniken“ im Lichte des technologischen Fortschritts festlegen sollten.

Eine automatisierte Überprüfung der Inhalte durch solche Inhaltserkennungstechniken könnte bedeuten, dass sogenannte Upload-Filter eingesetzt werden würden, d. h. ein Algorithmus, der Inhalte, die sich im Cache befinden, mit vorhandenen "Hashes“ ${ }^{\text {35 }}$ geschützter Werke vergleicht und gegebenenfalls aussortiert. ${ }^{36}$ Gewissermaßen kann man hier von einer Reaktion des Gesetzesgebers auf technologische Entwicklungen sprechen: das Hochladen und Verbreiten von Inhalten ist anhand digitaler Formate und globaler Netzwerke für jeden Internetnutzer möglich. Entsprechend wurde auch die Gegenmaßnahme aus der Perspektive gedacht, die Vermeidung von Rechteverletzungen zu automatisieren.

Der Einsatz von Upload-Filtern als Unterkategorie von Content-Control-Software ist grundsätzlich nicht neu: Digitale Plattformen, die nutzergenerierte Inhalte hosten, benutzen Filter seit geraumer Zeit bei solchen Inhalten, auf die sie anwendbar sind, vor allem bei audiovisuellen Inhalten. Sowohl die Forschung als auch die Rechtsprechung sind hier aufgrund negativer Effekte zurückhaltender: Beispielsweise hat eine Untersuchung der Software ContentID auf der Videoplattform YouTube gezeigt, dass viele Inhalte wegen einer vermeintlichen Verletzung von Urheberrechten entfernt wurden, obwohl dem nicht so war. ${ }^{37}$ Abgesehen von der Debatte rund um Ex-Art. 13 gibt es zwei Urteile des EuGH in denen das Gericht das verdachtslose Überprüfen von Informationen für rechtswidrig erklärt hat: SABAM/Netlog ${ }^{38}$ und Scarlet/SABAM. ${ }^{39}$ Eine Pflicht zur Einführung von automatisierter ex ante Überprüfung und Löschung von Inhalten wäre außerdem verfassungswidrig. ${ }^{40}$

35 Techterms, Hash, https://techterms.com/definition/hash (zuletzt abgerufen am 10.12.2018).

36 Heldt, in: Mohabbat-Kar/Thapa/Parycek, (Un)berechenbar? Algorithmen und Automatisierung in Staat und Gesellschaft, 392, 398.

37 Perel/Elkin-Koren, 181,197; Duarte u. a., Mixed Messages?.

38 EuGH, Urt. v. 16.2.2012 - C 360/10, GRUR 2012, 382 - SABAM v. Netlog NV.

39 EuGH, Urt. v. 24.11.2011 - C 70/10, GRUR 2012, 265 - Scarlet Extended v. SABAM.

40 Heldt, jipitec 2019, para 1; Spindler, Gutachten zur Urheberrechtsrichtlinie (DSM$\mathrm{RL})$. 


\subsection{Ergebnis: Wortlautwechsel}

Die Formulierung „wirksame Inhaltserkennungstechniken“ in Ex-Art. 13 stieß auf deutliche Kritik - in der öffentlichen Debatte ging es um das „Ende des Internets" durch Upload-Filter. ${ }^{41}$ Im Folgenden wurde der Passus gestrichen und die Bundesregierung erklärte sich bereit, in der deutschen Umsetzung der Richtlinie Upload-Filter „weitgehend unnötig zu machen. " $42 \mathrm{Ob}$ zwischen den Protesten und der Änderung eine sine qua non Kausalität festgestellt werden kann, lässt sich hier nicht abschließend klären, aber es gab durchaus kritische Reaktionen aus der Politik, ${ }^{43}$ eine Petition auf EU-Ebene ${ }^{44}$ und zahlreiche Versammlungen europaweit.

In der endgültigen Fassung des Art. 17 für die „Nutzung geschützter Inhalte durch Diensteanbieter für das Teilen von Online-Inhalten“ ist der Passus „wirksame Inhaltserkennungstechniken“ nicht enthalten. ${ }^{45}$ Stattdessen wird auf den Abschluss von Lizenzverträgen und die bestmöglichen Anstrengungen, die Erlaubnis des Rechtsinhabers einzuholen, abgestellt. Die Formulierung wurde geändert, aber anders als mit Upload-Filtern lässt sich die Pflicht aus Art. 17 Abs. 4 nicht einhalten. ${ }^{46}$ Die Haftungsprivilegierung des Art. 14 E-Commerce-Richtlinie wird bei einem Verstoß gegen Art. 17 aufgehoben - damit kommt es in puncto Haftung zu einer Ausnahme der Ausnahme. Ob die Gegenreaktionen auf den geplanten Ex-Art. 13 in Form von Versammlungen (mittlere Öffentlichkeitsebene) und kriti-

41 Reuter, Weitere Proteste gegen Urheberrechtsreform angekündigt, https://netzpoli tik.org/2019/weitere-proteste-gegen-urheberrechtsreform-angekuendigt/,(zuletzt abgerufen am 05.04.201); Reynolds, What is Article 13? The EU's divisive new copyright plan explained, https://www.wired.co.uk/article/what-is-article-13-article-1 1-european-directive-on-copyright-explained-meme-ban, (zuletzt abgerufen am 24.05.2019).

42 Bundesregierung, Protokollerklärung vom 15. April 2019: https://www.parlament. gv.at/PAKT/EU/XXVI/EU/06/18/EU_61832/imfname_10895457.pdf (zuletzt abgerufen am 20.6.2020).

43 Beispielsweise von den netzpolitischen Vereinen der Parteien CDU/CSU, SPD und FDP: D64/cnetz/Load, Offener Brief, https://www.load-ev.de/wp-content/uplo ads/2018/06/180629_Schreiben-EP_Uploadfilter_final.pdf, (zuletzt abgerufen am 12.08.2020).

44 Petition No 0424/2018 by P. Z. (Polish) on deleting Article 11 and Article 13 from the draft Directive on Copyright in the Digital Single Market.

45 Richtlinie (EU) 2019/790 des Europäischen Parlaments und des Rates v. 17.4.2019, https://eur-lex.europa.eu/legal-content/DE/TXT/HTML/?uri=CELEX:32 019L0790\&qid=1579079069995\&from=DE\#d1e1367-92-1, (zuletzt abgerufen am 15.1.2020).

46 Vgl. Spindler, CR 2019, 277, 285. 
scher Berichterstattung (massenmediale Öffentlichkeitsebene) zu einem Wendepunkt im Gesetzgebungsverfahren geführt haben, lässt sich nicht abschließend ermitteln. Die Verbreitung einer Information von der einfachen in die komplexe Ebene der Öffentlichkeit korrespondiert mit einer stärkeren Wahrnehmung des gesellschaftlichen Problems und erhöht somit die Zahl der individuellen Reaktionen hin zu einer kollektiven Reaktion, die auf einen Tipping Point hinweist. Ob Änderungen der öffentlichen Meinung zu einem Tipping Point führen, bleibt indes offen.

\section{Fazit}

Welcher Tipping Point war für die neue Urheberrechtsrichtlinie ausschlaggebend? Während des Gesetzgebungsverfahrens haben sich Machtverhältnisse an mindestens zwei Stellen verändert und Machtbeteiligungen verschoben: Diejenigen, die eine Novellierung der Richtlinie für notwendig erachtet haben und darin einen Gesetzgebungsauftrag gesehen haben, konnten eine kritische Zahl erreichen und führten somit zu einem Wendepunkt. Später fand ein Richtungswechsel in der Debatte statt, der maßgeblich Einfluss auf die Regelung zur Nutzung geschützter Inhalte hatte eine Verschiebung der Mehrheitsverhältnisse aufgrund der öffentlichen Debatte über Upload-Filter.

In der Diskussion um Tipping Points im Urheberrecht lohnt es sich ein genaueres Verständnis des Begriffs des Tipping Points und der Modelle, die dabei helfen sie zu identifizieren, heranzuziehen. Ausgehend von der Punctuated-Equilibrium-Theorie und dem Threshold-Modells lassen sich mögliche Zusammenhänge zwischen öffentlicher Debatte und Gesetzgebungsverfahren erkennen. Dabei wird klar, dass Themen, die auf unterschiedlichen Öffentlichkeitsebenen auf Resonanz stoßen, zu Reaktionen des Gesetzgebers führen können - auch wenn darin keine Reaktionspflicht zu sehen ist. 


\section{Literatur}

Baumgartner, Frank R. / Jones, Bryan D., Agendas and Instability in American Politics, 2. Ed., 2009.

Bundesregierung, Protokollerklärung vom 15. April 2019: https://www.parlament.gv .at/PAKT/EU/XXVI/EU/06/18/EU_61832/imfname_10895457.pdf (zuletzt abgerufen am 20.6.2020).

D64/cnetz/Load, Offener Brief, https://www.load-ev.de/wp-content/uploads/2018/06 /180629_Schreiben-EP_Uploadfilter_final.pdf, (zuletzt abgerufen am 12.08.2020).

Dan Wood, B. / Doan, Alesha, The Politics of Problem Definition: Applying and Testing Threshold Models, Am J Political Science 2003, S. 640-653.

Duarte, Natasha / Llanso, Emma / Loup, Anna, Mixed Messages? The Limits of Automated Social Media Content Analysis., FAT 2018, S. 106.

Fraenkel, Ernst, Demokratie und Öffentliche Meinung, Zeitschrift für Politik 1963, S. 309-328.

Gerhards, Jürgen, Öffentlichkeit, in: Jarren, Otfried/Sarnicelli, Ulrich/Saxer, Ulrich (Hrsg.), Politische Kommunikation in der demokratischen Gesellschaft: ein Handbuch mit Lexikonteil, 1. Aufl., Opladen 1998, S. 268-284.

Gerhards, Jürgen / Neidhardt, Friedhelm, Strukturen und Funktionen moderner Öffentlichkeit: Fragestellungen und Ansätze, 1990, S. 60.

Gielen, Nico / Tiessen, Marten, Die neue Plattformhaftung nach der Richtlinie über das Urheberrecht im digitalen Binnenmarkt, EuZW 2019, S. 639-646.

Gladwell, Malcolm, Der Tipping Point: wie kleine Dinge Grosses bewirken können, Berlin 2000.

Granovetter, Mark, Threshold Models of Collective Behavior, AJS 1978, 1420-1443.

Habermas, Jürgen, Strukturwandel der Öffentlichkeit: Untersuchungen zu einer Kategorie der bürgerlichen Gesellschaft; mit einem Vorwort zur Neuauflage 1990, 1. Aufl, Frankfurt am Main 1990.

Heldt, Amélie, Intelligente Upload-Filter: Bedrohung für die Meinungsfreiheit?, in: Mohabbat-Kar, Resa / Thapa, Basanta E.P / Parycek, Peter (Hrsg.), (Un)berechenbar? Algorithmen und Automatisierung in Staat und Gesellschaft, Berlin 2018, S. 392-416.

Heldt, Amélie Pia, Upload-Filters: Bypassing Classical Concepts of Censorship?, jipitec 2019, para 1.

Hoffmann-Riem, Wolfgang/Schulz, Wolfgang, Politische Kommunikation - Rechtswissenschaftliche Perspektiven, in: Jarren, Otfried /Sarcinelli, Ulrich /Saxer, Ulrich (Hrsg.), Politische Kommunikation in der demokratischen Gesellschaft: Ein Handbuch mit Lexikonteil, Wiesbaden 1998, S. 154-172.

Lüdemann, Christian, Gesetzgebung als Entscheidungsprozess: zur Normgenese der strafrechtlichen Regelung zur Strafaussetzung bei lebenslanger Freiheitsstrafe, Opladen 1986. 
Noelle-Neumann, Elisabeth, Öffentliche Meinung: die Entdeckung der Schweigespirale, Erw. Ausg., Frankfurt/Main [u.a.] 1991.

Perel, Maayan / Elkin-Koren, Niva, Black Box Tinkering: Beyond Disclosure in Algorithmic Enforcement, Fla. L. R. 2017, S. 181-221.

Pfetsch, Barbara/ Löblich, Maria/ Eilders, Christiane, Dissonante Öffentlichkeiten als Perspektive kommunikationswissenschaftlicher Theoriebildung, Publizistik 2018, 477-495.

Popitz, Heinrich, Die normative Konstruktion von Gesellschaft, Tübingen 1980.

Reuter, Markus, Weitere Proteste gegen Urheberrechtsreform angekündigt, https:// netzpolitik.org/2019/weitere-proteste-gegen-urheberrechtsreform-angekuendigt/, (zuletzt abgerufen am 05.04.201).

Reynolds, Matts, What is Article 13? The EU's divisive new copyright plan explained, (https:/www.wired.co.uk/article/what-is-article-13-article-11-european-d irective-on-copyright-explained-meme-ban zuletzt abgerufen am 24.05.2019).

Techterms, Hash, https://techterms.com/definition/hash (zuletzt abgerufen am 10.12.2018).

Schmitt, Carl, Verfassungslehre, 8., unveränd. Aufl., Berlin 1993.

Spindler, Gerald, Die neue Urheberrechts-Richtlinie der EU, insbesondere „UploadFilter“ - Bittersweet?, CR 2019, S. 277-291.

Spindler, Gerald, Gutachten zur Urheberrechtsrichtlinie (DSM-RL): Europarechtliche Vereinbarkeit (Artikel 17), Vorschläge zur nationalen Umsetzung und zur Stärkung der Urheberinnen und Urheber, 2019, S. 73.

Wandtke, Artur-Axel, Urheberrecht in der Reform oder wohin steuert das Urheberrecht? MMR (2017), S. 367. 